\title{
Numerical Approach of Linear Volterra Integro-Differential Equations Using Generalized Spline Functions
}

\author{
Nabaa N. Hasan* \\ Received 13, November, 2011 \\ Accepted 30, January, 2012
}

\begin{abstract}
:
This paper is dealing with non-polynomial spline functions "generalized spline" to find the approximate solution of linear Volterra integro-differential equations of the second kind and extension of this work to solve system of linear Volterra integrodifferential equations. The performance of generalized spline functions are illustrated in test examples.
\end{abstract}

Keywords: Integro-differential equations, generalized spline functions.

\section{Introduction:}

Integro-differential equation (IDE) is an important branch of modern Mathematics and arises frequently in many applied areas which include engineering, mechanics, Physics, Chemistry, Biology, economics and electrostatics, [1]. Authors and researchers used numerical methods to find a solution of the integrodifferential equations. Elayaraja and Jumat, in [2], Generalized Minimal Residual (GMR) method may be used to approximate the solution of linear Fredholm integro-differential equation of second order which discretized by using finite difference and trapezoidal methods. Rostam and Kawa, in [3], deal with introducing spline function to find the approximate solution of nonlinear fredholm integral equations. Mortaza, in [4], presented Power series method for finding the numerical solution of linear and nonlinear integro-differential equations system. Muna in, [5], proposed numerical methods to solve Volterra integral equations and state theorem which reduce high order system to first order for linear Volterra integro-differential equations. In this papear, generalized spline functions will be used for solving the following two problems:

- Linear Volterra integro-differential equation (VIDE) of $\mathrm{n}^{\text {th }}$ order :

$\left(D^{n}+\sum_{i=0}^{n-1} p_{i}(x) D^{i}\right) u(x)=f(x)+\int_{a}^{x} k(x, t) u(t) d t, x \in[a, b]--(1)$ with initial conditions $u(a), u^{\prime}(a), \ldots, u^{n-1}(a)$ are be given, where, $\mathrm{f}, \mathrm{p}_{\mathrm{i}}$ and $\mathrm{k}$ denote given continuous functions.

- System of the $1^{\text {st }}$ order linear Volterra integro-differential equation (VIDE's):

$$
\begin{aligned}
& \left(D+p_{i}(x)\right) u_{i}(x)=f_{i}(x)+\sum_{j=1}^{m} \int_{a}^{x} k_{i j}(x, t) u_{j}(t) d t \\
& ; x \in I=[a, b], i=1,2, \ldots, m \quad---(2)
\end{aligned}
$$

with the initial conditions: $u_{i}(a)=u_{i}$ $; i=1,2, \ldots, m$

where the functions $f_{i}$, and $p_{i}$; $\mathrm{i}=1,2, \ldots, \mathrm{m}$ are assumed to be continuous on $I$ and $k_{i, j} ; i, j=1,2, \ldots, m$ denotes some given continuous functions.

Generalized Splines, [6]

Consider the linear differential operator of order $\mathrm{p} \in N$

$L=D^{p} .+a_{p-1}(t) D^{p-1} .+\ldots+a_{1}(t) D .+a_{0}(t) .---(3)$

where each $a_{k}(t), k=0,1, \ldots, p-1$, is a 
real $C^{p}$ smooth function in $[\mathrm{a}, \mathrm{b}]$. The operator $L$ is acting on the space $C^{m}[a, b]$ of real functions defined in [a,b]. Its adjoint is defined by

$L^{*}=(-1)^{p} D^{p} \cdot+(-1)^{p-1} D^{p-1}\left(a_{p-1}(t) \cdot\right)+\ldots-D\left(a_{1}(t).\right)+a_{0}(t) \cdot---(4)$

$L^{*}$ is also acting on the space $C^{m}[a, b]$ and the scalar product for which it is computed is given by

$$
\left\langle x_{1}, x_{2}\right\rangle=\int_{a}^{b} x_{1}(t) x_{2}(t) d t
$$

Let

$a=t_{0}<t_{1}<\ldots<t_{m}=b, m \in N$, be a partition of $[a, b], \Omega$ be the family of real $C^{2 p-2}$ smooth functions in $[a, b]$ which are $C^{2 p}$ smooth in each interval $\left[t_{i}, t_{i+1}\right], i=0,1, \ldots, m-1$ and $f \in \Omega$.

Definition (2.1), [7]:]

The function $\mathrm{s}:[a, b] \rightarrow \mathrm{R}$ is an interpolating generalized spline of $f$ associated to $\Delta$ and $L$, if $s \in \Omega$, s is a solution of the differential equation $L^{*} L \quad \mathrm{x}=0$ in each interval $\left[t_{i}, t_{i+1}\right], i=0,1, \ldots, m-1$, and $s(t)=f(t)$ on $\Delta$.

Definition (2.2), [7]:

An interpolating generalized spline of $f$ is of type $\mathbf{I}$ if it is such that $s^{(k)}\left(t_{0}\right)=f^{(k)}\left(t_{0}\right) \quad$ and $s^{(k)}\left(t_{m}\right)=f^{(k)}\left(t_{m}\right)$ for $k=1,2, \ldots, p-1$ By interpolation and boundary conditions gave in definitions $(2,1)$ and (2.2) respectively $s$ is a generalized spline of type $\mathbf{I}$.

Now to construct the approximate function, let $q_{j}, j=1,2, \ldots, 2 h$, be the basis functions of generalized spline $s$, where $2 h$ is order of $L^{*} L \mathrm{x}=0$ gave in definition $(2,1)$.Then generalized spline functions $s$ is defined by:

$s(t)=\sum_{j=1}^{2 h} c_{j} q_{j}(t)$

In each

$\left[t_{i}, t_{i+1}\right], i=0,1, \ldots, m-1$

interval

where

$c_{1}, c_{2}, \ldots, c_{2 h}$ are constants to be found.
Particularly consider the operator of order $2^{\text {nd }}$ given by:

$L=D^{2} .-4$ then its adjoint is $L^{*}=D^{2} .-4$ by solving the homogeneous differential equation $L^{*} L=D^{4} .-8 D^{2} .+16$, we have the solutions: $e^{2 t}, e^{-2 t}, t e^{2 t}, t e^{-2 t}$

so that the generalized spline function in each $\left[t_{i}, t_{i+1}\right]$, is:

$s(t)=c_{1} e^{2 t}+c_{2} e^{-2 t}+c_{3} t e^{2 t}+c_{4} t e^{-2 t}$

\section{Approximate Solution of $\mathbf{n}^{\text {th }}$ Order Linear VIDE}

In this section the reduction theorem, [8] will be used to reduce the integrodifferential equations of arbitrary high order to the first order in which one can use the available spline function to solve eq.(1).

Theorem (1.3): (The Reduction Theorem),[8]

Let $\mathrm{f}, \mathrm{k}$ be $\mathrm{L}^{2}$ integrable functions on interval $[a, b]$ and $p_{i} \in C^{n}[a, b]$. Then eq.(1) may be reduced to the first order linear VIDE of the form:

$\left(D+p_{n-1}(x)\right) u(x)=F_{n-1}(x)+\int_{a}^{x} k_{n-1}(x, t) u(t) d t$

with initial condition:

$\mathrm{u}(a)=\mathrm{u}_{0}$

where

$F_{n-1}(x)=\sum_{i=1}^{n-1} \frac{u_{i} x^{i-1}}{(i-1) !}+\frac{1}{(n-2) !} \int_{a}^{x}(x-t)^{n-2} f(t) d t+$

$\sum_{k=0}^{n-2} \sum_{j=k+1}^{n-1} \sum_{=n+k-j}^{n-1}(-1)^{k} p_{j}^{(k)}(a) A_{k, n-i-1} \frac{u_{i+j-n-k}}{(i-1) !}$ and

$k_{n-1}(x, t)=\frac{1}{(n-2) !} \int_{t}^{x}\left(x-z_{1}\right)^{n-2} k\left(z_{1}, t\right) d z_{1}-$

$\sum_{k=0}^{n-2} \sum_{j=0}^{k+1}(-1)^{j} p_{j+n-k-2}^{(j)} B_{n-k-2, j} \frac{(x-t)^{k}}{k !}$

with $[\mathrm{A}]$ and $[\mathrm{B}]$ being two special constant matrices of dimensions (n$2) \times(n-2)$ and $(n-1) \times(n-1)$ respectively.

For more details and proof about reduction theorem see [8]

To find the approximate solution of eq.(1), substitute eq.(5) in eq.(7) we get:

$\left(D+p_{n-1}(x)\right) \sum_{j=1}^{2 h} c_{j} q_{j}(x)-\int_{a}^{x} k_{n-1}(x, t) \sum_{j=1}^{2 h} c_{j} q_{j}(t) d t=F_{n-1}(x)$ 
$\sum_{j=1}^{2 h} c_{j}\left[\left(D+p_{n-1}(x)\right) q_{j}(x)-\int_{a}^{x} k_{n-1}(x, t) q_{j}(t) d t\right]=F_{n-1}(x)$

Let

$M_{j}(x)=\left[\left(D+p_{n-1}(x)\right) q_{j}(x)-\int_{a}^{x} k_{n-1}(x, t) q_{j}(t) d t\right.$

$, j=1,2, \ldots, 2 h$

where $\mathrm{x}=\mathrm{x}_{\mathrm{r}}, r=0,1, \ldots, N$, and adding the initial condition of eq.(9) as a new raw then we can write eq.(10) as in the following system:
$A=\left(\begin{array}{cccc}M_{1}\left(x_{0}\right) & M_{2}\left(x_{0}\right) & \cdots & M_{2 h}\left(x_{0}\right) \\ M_{1}\left(x_{1}\right) & M_{2}\left(x_{1}\right) & \cdots & M_{2 h}\left(x_{1}\right) \\ & \cdots & \cdots & \cdots \\ M_{1}\left(x_{N}\right) & M_{2}\left(x_{N}\right) & \cdots & M_{2 h}\left(x_{N}\right) \\ q_{1}(a) & q_{2}(a) & \cdots & q_{2 h}(a)\end{array}\right), \quad C=\left(\begin{array}{c}c_{1} \\ c_{2} \\ \vdots \\ c_{2 h}\end{array}\right), F=\left(\begin{array}{c}F_{n-1}\left(x_{0}\right) \\ F_{n-1}\left(x_{1}\right) \\ \vdots \\ F_{n-1}\left(x_{N}\right) \\ u_{0}\end{array}\right)---(9)$

or

$A C=F$

$A$ and $F$ are constant matrices with dimensions $(N+2) \times 2 h$ and $(N+2) \times 1$ respectively. Calculate $A^{T} A C=A^{T} F$ to find the $c_{j} ; j=1,2, \ldots, 2 h$ and substitute this solution in eq.(5) to get the approximate solution of eq.(1).

Approximate Solution of System of Linear VIDE

In this section the system of the $1^{\text {st }}$ order linear VIDE's given in eq.(2) will be formulated in matrix form, as:

$$
\begin{gathered}
\left(D+p_{2}(x)\right) u_{2}(x)-\int_{a}^{x} k_{22}(x, t) u_{2}(t) d t=\int_{a}^{x} k_{21}(x, t) u_{1}(t) d t+\ldots+\int_{a}^{x} k_{2 m}(x, t) u_{m}(t) d t \\
\left(D+p_{m}(x)\right) u_{m}(x)-\int_{a}^{x} k_{m m}(x, t) u_{m}(t) d t=\int_{a}^{x} k_{m 1}(x, t) u_{1}(t) d t+\ldots+\int_{a}^{x} k_{m, m-1}(x, t) u_{m-1}(t) d t
\end{gathered}
$$

then:

$\mathrm{AC}=\mathrm{F}$

where $A=\left(a_{i, j}\right)$ as a matrix of order $m$ such that

$$
a_{i j}=\sum_{i=1}^{m} k_{i j} u_{j} \quad i \neq j ; i, j=1,2, \ldots, m
$$$$
a_{i i}=\left(D+p_{i}(x)\right) u_{i}(x)-\int_{a}^{x} k_{i i}(x, t) u_{i}(t) d t
$$$$
; i=1,2, \ldots, m
$$

$$
C=\left[c_{11}, c_{12}, \ldots, c_{1,2 h}, c_{21}, c_{22}, \ldots, c_{2,2 h}, \ldots, c_{m 1}, c_{m 2}, \ldots, c_{m, 2 h}\right]
$$

$$
F=\left[f_{1}, f_{2}, \ldots, f_{m}\right]
$$

\section{Algorithm(1)}

Step(1): Input $N$ (number of subintervals). Set $h 1=\frac{b-a}{N}$, $\mathrm{x}_{\mathrm{r}}=a+\mathrm{r} h 1$, for $\mathrm{r}=0,1, \ldots, N$ and divide the interval [a,b] into $N$ subintervals, $a=x_{0}\left\langle x_{1}\left\langle\ldots<x_{N}=b\right.\right.$.

Step(2): let $s_{i}(a)=u_{i} \quad ; i=1,2, \ldots, m$ (which are the given initial conditions ).
Step(3): let $\quad s_{i}(x)=\sum_{j=1}^{2 h} c_{i j} q_{j}(x) \quad$;

$$
i=1,2, \ldots, m
$$

Step(4): using step(3) and eq.(11) to find the coefficients $c_{i j}, i=1,2, \ldots, m$; $j=1,2, \ldots, 2 h$.

Step(5): substitute the results in step(4) to get the approximation solution of eq.(2).

\section{Numerical Examples:}

Three numerical test examples were defined on the interval $[0,1]$ and 
$\Delta: 0=x_{0}<x_{1}<x_{2}<x_{3}=1$, where

$h=\frac{1}{3}$, for examples (5.1) and

apply generalized spline function defined in eq. (6) and for example (5.3) apply generalized spline function defined in step (3) of algorithm (1)

\section{Example(5.1):}

Consider the $1^{\text {st }}$ order linear VIDE problem

$$
(D+\cos (x)) u(x)=\cos (x) e^{x}+x+1+\int_{0}^{x}(x-t) u(t) d t
$$

, $0 \leq x \leq 1$

with initial condition:

$\mathrm{u}(0)=1$

The exact solution is:

$u(x)=e^{x}$

In eq.(6) the coefficients $c_{1}, c_{2}, c_{3}, c_{4}$

are unknown, four algebraic equations are needed. substituting eq.(6) in the initial condition eq.(13) yield:

$$
c_{1}+c_{2}+c_{3}+c_{4}=1
$$

substituting eq.(6) in eq.(12) for each $x=x_{i}, i=0,1,2,3$ yield:

$$
\begin{array}{ll}
2 c_{1} e^{2 x}-2 c_{2} e^{-2 x}+c_{3}(1+2 x) e^{2 x}+c_{4}(1-2 x) e^{-2 x}+\cos x\left(c_{1} e^{2 x}+c_{2} e^{-2 x}+c_{3} x e^{2 x}+c_{4} x e^{-2 x}\right)- \\
\int_{0}^{x}(x-t)\left(c_{1} e^{2 t}+c_{2} e^{-2 t}+c_{3} t e^{2 t}+c_{4} t e^{-2 t}\right) d t=f(x)-(15) & \text { where } A \text { is constant matrix of } \\
\text { where } & \text { dimension } \quad(5 \times 4) \text { gain from eq. (14) } \\
f(x)=\cos (x) e^{x}+x+1 & \text { and eq.(15) } \quad C=\left[\begin{array}{lllll}
c_{1} & c_{2} & c_{3} & c_{4}
\end{array}\right]^{T} \\
\text { The system will construct from eq.(14) } & F=\left[\begin{array}{lllll}
u(0) & f\left(x_{0}\right) & f\left(x_{1}\right) & f\left(x_{2}\right)
\end{array}\right.
\end{array}
$$
and eq.(15) has 5 equations and 4 coefficients, therefore, calculate:

$$
A^{T} A C=A^{T} F
$$

Finally, Gauss elimination method may be used to solve system (16) to find $c_{1}=0.725, c_{2}=0.275, c_{3}=-0.371$, $c_{4}=0.469$, so the approximate solution $s(x)$ is:

$$
s(x)=0.725 e^{2 x}+0.275 e^{-2 x}-0.371 x e^{2 x}+0.469 x e^{-2 x}
$$

Table(1), presents a comparison between the exact and numerical solution for $u(x)$.

Table(1): Numerical Results of Example (5.1)

\begin{tabular}{|c|c|c|c|}
\hline $\mathrm{x}$ & exact & approximate & absolute error \\
\hline 0 & 1 & 1 & 0 \\
\hline 0.1 & 1.10517091 & 1.10375238 & 0.00141853 \\
\hline 0.2 & 1.22140275 & 1.21809354 & 0.00330921 \\
\hline 0.3 & 1.34985880 & 1.34637530 & 0.00348350 \\
\hline 0.4 & 1.49182469 & 1.49110647 & 0.00071821 \\
\hline 0.5 & 1.64872127 & 1.65394762 & 0.00522635 \\
\hline 0.6 & 1.82211880 & 1.83561120 & 0.01349240 \\
\hline 0.7 & 2.01375270 & 2.03565649 & 0.02190378 \\
\hline 0.8 & 2.22554092 & 2.25216160 & 0.02662067 \\
\hline 0.9 & 2.45960311 & 2.48124697 & 0.02164386 \\
\hline 1 & 2.71800000 & 2.71986700 & 0.001867 \\
\hline
\end{tabular}

As a cooperation the least square error is $5.158 \times 10^{-7}$ while the partition method gave in [9], is $9 \times 10^{-6}$.

\section{Example(5.2):}

Consider the $4^{\text {th }}$ order linear VIDE problem

$\left(D^{4}-D^{3}+D^{2}-D-x^{2}\right) u(x)=24-x^{4}+\frac{19}{30} x^{6}+\frac{29}{210} x^{7}+\int_{0}^{x}(x+t) u(t) d t$

with initial conditions:

$$
u(0)=u^{\prime}(0)=u^{\prime \prime}(0)=u^{\prime \prime \prime}(0)=0
$$

The exact solution is: 


$$
u(x)=x^{4}+\frac{x^{5}}{5}
$$

By using the reducing theorem we have the following $1^{\text {st }}$ order linear VIDE problem:

$$
(D-1) u(x)=F_{3}(x)+\int_{0}^{x} k_{3}(x, t) u(t) d t
$$

where

$$
F_{3}(x)=4 x^{3}-\frac{x^{7}}{210}+\frac{19}{15120} x^{9}+\frac{29}{151200} x^{10}
$$

$k_{3}(x, t)=\frac{x^{4}}{24}+\frac{x^{3} t}{6}-\frac{5}{4} x^{2} t^{2}+\frac{11}{6} x t^{3}-\frac{19}{24} t^{4}+x-t-1$

with initial conditions

$$
u(0)=0
$$

As in example(5.1) Gauss elimination method may be used to solve system (16) for eq.(17) and eq.(18) to find $c_{1}=-0.296, c_{2}=0.292, c_{3}=0.436$, $c_{4}=0.748, \quad$ so the approximate solution

is:

$$
s(x)=-0.296 e^{2 x}+0.292 e^{-2 x}+0.436 x e^{2 x}+0.748 x e^{-2 x}
$$

Table(2), presents a comparison between the exact and numerical solution for $u(x)$.

Table(2): Numerical Results of Example (5.2)

\begin{tabular}{|c|c|c|c|}
\hline $\mathrm{x}$ & exact & approximate & absolute error \\
\hline 0 & 0 & 0.00400000 & 0.00400000 \\
\hline 0.1 & 0.000102 & -0.00797161 & 0.00807361 \\
\hline 0.2 & 0.001664 & -0.01547966 & 0.01714366 \\
\hline 0.3 & 0.008586 & -0.01760769 & 0.02619369 \\
\hline 0.4 & 0.027648 & -0.00498249 & 0.03263049 \\
\hline 0.5 & 0.068759 & 0.03298172 & 0.03576827 \\
\hline 0.6 & 0.145152 & 0.10891265 & 0.03623934 \\
\hline 0.7 & 0.273714 & 0.23843232 & 0.03528167 \\
\hline 0.8 & 0.475136 & 0.44128877 & 0.03384722 \\
\hline 0.9 & 0.774198 & 0.74273250 & 0.03146549 \\
\hline 1 & 1.200000 & 1.17500000 & 0.02500000 \\
\hline
\end{tabular}

\section{Example(5.3):}

Consider the system of linear VIDE problem

$$
\begin{aligned}
& D u_{1}(x)=1+x+x^{2}-u_{2}(x)-\int_{0}^{x}\left(u_{1}(t)+u_{2}(t)\right) d t \\
& 0 \leq x \leq 1 \quad--(19) \\
& D u_{2}(x)=-1-x+u_{1}(x)-\int_{0}^{x}\left(u_{1}(t)-u_{2}(t)\right) d t, \\
& 0 \leq x \leq 1 \quad---(20)
\end{aligned}
$$

with initial conditions:

$$
\begin{aligned}
& u_{1}(0)=1, \\
& u_{2}(0)=-1,
\end{aligned}
$$

The exact solutions are:

$$
\begin{gathered}
u_{1}(x)=x+e^{x} \\
u_{2}(x)=x-e^{x}
\end{gathered}
$$

In this example as in step(3), algorithm(1), $u_{1}(x)$ will approximate by $s_{1}(x)$, where

$$
s_{1}(x)=c_{11} e^{2 x}+c_{12} e^{-2 x}+c_{13} x e^{2 x}+c_{14} x e^{-2 x}--(23)
$$

and $u_{2}(x)$ will approximate by $s_{2}(x)$, where

$$
s_{2}(x)=c_{21} e^{2 x}+c_{22} e^{-2 x}+c_{23} x e^{2 x}+c_{24} x e^{-2 x}
$$

To find the unknown coefficients $c_{i j}, i=1,2 ; j=1,2,3,4$, eight algebraic equations are needed. Substituting eq.(23) in the initial condition eq.(21) yield:

$$
\begin{aligned}
& c_{11}+c_{12}+c_{13}+c_{14}=1 \quad \text { each } x=x_{i}, i=0,1,2,3 \text { yield: } \\
& 2 c_{11} e^{2 x}-2 c_{12} e^{-2 x}+c_{13}(1+2 x) e^{2 x}+c_{14}(1-2 x) e^{-2 x}+\left(c_{21} e^{2 x}+c_{22} e^{-2 x}+c_{23} x e^{2 x}+c_{24} x e^{-2 x}\right)+ \\
& \int_{0}^{x}\left(c_{11} e^{2 t}+c_{12} e^{-2 t}+c_{13} t e^{2 t}+c_{14} t e^{-2 t}+c_{21} e^{2 t}+c_{22} e^{-2 t}+c_{23} t e^{2 t}+c_{24} t e^{-2 t}\right) d t=f_{1}(x)-(27)
\end{aligned}
$$

and eq.(24) in the initial condition eq.(22) yield:

$c_{21}+c_{22}+c_{23}+c_{24}=-1$

substituting eq.(23) and eq.(24) in eq.(19) and eq.(20) respectively for 


$$
\begin{aligned}
& 2 c_{21} e^{2 x}-2 c_{22} e^{-2 x}+c_{23}(1+2 x) e^{2 x}+c_{24}(1-2 x) e^{-2 x}-\left(c_{11} e^{2 x}+c_{12} e^{-2 x}+c_{13} x e^{2 x}+c_{14} x e^{-2 x}\right)+ \\
& \int_{0}^{x}\left(c_{11} e^{2 t}+c_{12} e^{-2 t}+c_{13} t e^{2 t}+c_{14} t e^{-2 t}-c_{21} e^{2 t}-c_{22} e^{-2 t}-c_{23} t e^{2 t}-c_{24} t e^{-2 t}\right) d t=f_{2}(x)---(28) \\
& \text { where } \quad \begin{array}{r|r|r|r|}
\hline 0.9 & 3.35960311 & 3.54475599 & 0.185152880 \\
\hline 1 & 3.71800000 & 3.85200000 & 0.13400000 \\
\hline
\end{array}
\end{aligned}
$$

$$
\begin{aligned}
& f_{1}(x)=1+x+x^{2} \\
& f_{2}(x)=-1-x
\end{aligned}
$$

The system will construct from eqs.(25)-(28) has 10 equations and 8 coefficients, therefore, calculate:

$$
A^{T} A C=A^{T} F
$$

where $A$ is constant matrix of dimension $(10 \times 8)$ gain from the eqs.(25)-(28) and $C=\left[\begin{array}{lll}c_{11} & c_{12} & c_{13}\end{array}\right.$ $\left.c_{14} c_{21} \quad c_{22} \quad c_{23} c_{24}\right]^{T}$

$F=\left[\begin{array}{llll}u_{1}(0) & u_{2}(0) & f_{1}\left(x_{0}\right) & f_{2}\left(x_{0}\right)\end{array}\right.$ $f_{1}\left(x_{1}\right) \quad f_{2}\left(x_{1}\right) \quad f_{1}\left(x_{2}\right) \quad f_{2}\left(x_{2}\right) \quad f_{1}\left(x_{3}\right)$ $\left.f_{2}\left(x_{3}\right)\right]^{T}$

Finally, Gauss elimination method may be used to solve system (29) to find $c_{11}=1.011$, $c_{12}=-0.011$, $c_{13}=-0.484$, $c_{14}=0.441$, $c_{21}=-0.389$, $c_{22}=-0.611$, $c_{23}=0.178, \quad c_{24}=-0.621, \quad$ so the approximate solutions $s_{1}(x)$ and $s_{2}(x)$ are:

$s_{1}(x)=1.011 e^{2 x}-0.011 e^{-2 x}-0.484 x e^{2 x}+0.441 x e^{-2 x}$

$s_{2}(x)=-0.389 e^{2 x}-0.611 e^{-2 x}+0.178 x e^{2 x}-0.621 x e^{-2 x}$

Table(3), and table(4), present a comparison between the exact and numerical solution for $\mathrm{u}_{1}(\mathrm{x})$ and $\mathrm{u}_{2}(\mathrm{x})$ respectively.

Table(3): Numerical Results of Eexample (5.3)

\begin{tabular}{|c|c|c|c|}
\hline $\mathrm{x}$ & exact & approximate & absolute error \\
\hline 0 & 1 & 1 & 0 \\
\hline 0.1 & 1.20517091 & 1.20282228 & 0.00234863 \\
\hline 0.2 & 1.42140275 & 1.41557484 & 0.00582791 \\
\hline 0.3 & 1.64985880 & 1.64416130 & 0.00569749 \\
\hline 0.4 & 1.89182469 & 1.89347616 & 0.00165146 \\
\hline 0.5 & 2.14872127 & 2.16742946 & 0.01870819 \\
\hline 0.6 & 2.42211880 & 2.46885910 & 0.04674030 \\
\hline 0.7 & 2.71375270 & 2.79931733 & 0.08556462 \\
\hline 0.8 & 3.02554092 & 3.15870985 & 0.13316892 \\
\hline
\end{tabular}

\begin{tabular}{|c|c|c|c|}
\hline 0.9 & 3.35960311 & 3.54475599 & 0.185152880 \\
\hline 1 & 3.71800000 & 3.85200000 & 0.13400000 \\
\hline
\end{tabular}

Table(4): Numerical Results of Example (5.3)

\begin{tabular}{|c|c|c|c|}
\hline $\mathrm{x}$ & exact & approximate & absolute error \\
\hline 0 & -1 & -1 & 0 \\
\hline 0.1 & -1.00517091 & -1.00447237 & 0.00069854 \\
\hline 0.2 & -1.02140275 & -1.02003014 & 0.00137261 \\
\hline 0.3 & -1.04985880 & -1.04907058 & 0.00078822 \\
\hline 0.4 & -1.09182469 & -1.09343021 & 0.00160552 \\
\hline 0.5 & -1.14872127 & -1.15448545 & 0.00576418 \\
\hline 0.6 & -1.22211880 & -1.23319162 & 0.01107282 \\
\hline 0.7 & -1.31375270 & -1.33006131 & 0.01630860 \\
\hline 0.8 & -1.42554092 & -1.44507875 & 0.01953782 \\
\hline 0.9 & -1.55960311 & -1.57754250 & 0.01793939 \\
\hline 1 & -1.71800000 & -1.72600000 & 0.00800000 \\
\hline
\end{tabular}

The method in [4], defines the error between the exact solution and the Taylor polynomial solution by the reminder $O\left(x^{7}\right)$.

\section{Conclusions:}

In this paper, the researcher have constructed generalized spline functions for solving linear VIDE's of $\mathrm{n}^{\text {th }}$ order and system of linear VIDE's based on interpolation and boundary conditions of the generalized spline. The choice of the linear differential operator gave the basis which form the approximate function. Three test examples are considered, the first deal with $1^{\text {th }}$ order linear VIDE's, the second reduce $4^{\text {th }}$ order linear VIDE's to $1^{\text {th }}$ order by reduction theorem, and the third solve system of linear first order VIDE's. As a comparison with the exact solution, tables (1), (2), (3), and (4) showed the results. Comparison with methods in references [9] and [4] are given.

\section{References:}

1. Oladejo S. O. and Olurode K.A. 2008. "The Application of Cubic Spline Collocation to the Solution 
of Integral Equations", J. Applied Sciences Research, 4(6):748-753.

2. Elayaraja Aruchunan and Jumat Sulaiman, 2010. "Numerical Solution of Second-Order Linear Fredholm Integro-Differential Equation Using Generalized Minimal Residual Method", J. American Applied Sciences, 7(6): 780-783.

3. Rostam K. Saeed and Kawa M.Aziz, 2008. "Approximate Solution of the System of Nonlinear Fredholm Integral Equations of the Second Kind Using Spline Functione", Journal of Kirkuk UniversityScienific Studies, 3(1): 113-126.

4. Mortaza Gachpazan, 2009. "Numerical Scheme to Solve Integro-Differential Equations System", Journal of Advanced Research in Scientific Computing, 1: 11-21.
5. Muna Mansur, 2004. "Numerical Solutions for System of Volterra Integral Equations Using Splines Function", Ph.D. Thesis, AlMustansiriya University.

6. Ahlberg J. H., Nilson E. N., Walsh J. L. 1967. "The Theory of Splines and Their Applications", Academic Press, New York.

7. Rui C. Rodrigues and Delfim F. M. Torres, 2006. "Generalized Splines in $\mathrm{R}^{\mathrm{n}}$ and Optimal Control", 64(1): 1-18.

8. Shazad S. Ahmed, 2002. "Numerical Solutions of Linear Volterra Integro-Differential Equations", M.Sc. Thesis, University of Technology.

9. Rabha S. Kareem, 2003. "Approximated Treatment of Higher-Order Linear Volterra Integro-Differential Equations", M.Sc. Thesis, University of Technology.

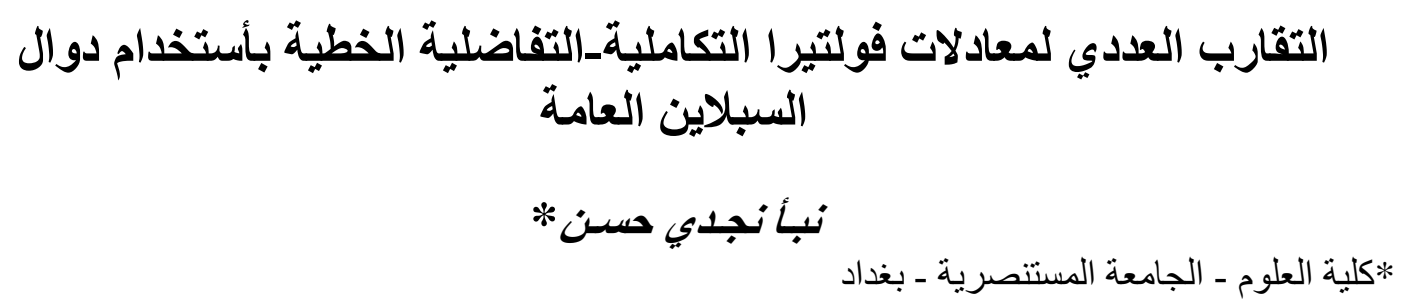

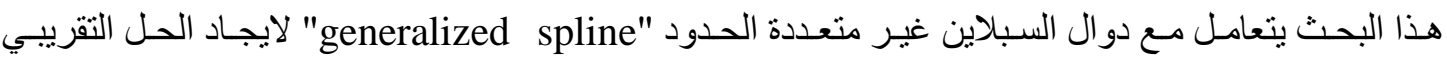

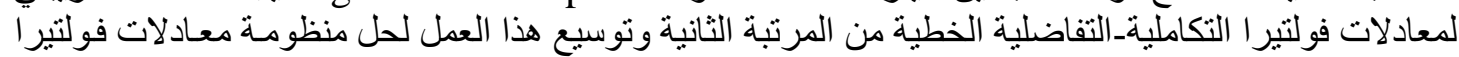
التكاملية_التفاضلية الخطية. أداء ودقة الطريقة موضحة من خلافل بالامثلة. 